\title{
CATÁLOGO DOS CECIDOMYIIDAE (DIPTERA) DO ESTADO DO RIO DE JANEIRO
}

\author{
Valéria Cid Maia ${ }^{1}$
}

Biota Neotropica v5 (n2) - http://www.biotaneotropica.org.br/v5n2/pt/abstract?inventory+BN00805022005

Recebido em 10/12/04.

Revisado em 16/04/05.

Publicado em 01/07/2005.

${ }^{1}$ Museu Nacional, Quinta da Boa Vista, São Cristóvão, Rio de Janeiro, RJ, Brasil. CEP: 20940-040
E-mail: maiavcid@acd.ufrj.br

\begin{abstract}
About 500 species of 170 genera of the Cecidomyiidae are known in the Neotropical Region. The cecidomyiids are poorly known in Brazil, where 159 named species of 75 genera have been reported. Most of the species known in Brazil have been reported from the State of Rio de Janeiro where 95 species of 47 genera have been recorded. The main goal of this paper is to contribute to the knowledge of the diversity of the cecidomyiids in the State of Rio de Janeiro.
\end{abstract}

Key words: Cecidomyiidae, Diptera, Insecta.

\section{RESUMO}

Cerca de 500 espécies de 170 gêneros de Cecidomyiidae são conhecidos da Região Neotropical. Os cecidomiídeos são pouco conhecidos no Brasil, onde 159 espécies de 75 gêneros têm sido relatadas. A maioria das espécies conhecidas do Brasil foram encontradas no Estado do Rio de Janeiro, onde 95 espécies de 47 gêneros têm sido registradas. O principal objetivo deste trabalho é contribuir para o conhecimento da diversidade dos cecidomiídeos no Estado do Rio de Janeiro.

Palavras-chave: Cecidomyiidae, Diptera, Insecta.

http://www.biotaneotropica.org.br 


\section{INTRODUÇÃO}

Cecidomyiidae é uma família bastante diversificada de Nematocera (Diptera). Os insetos desta família são encontrados em todas as regiões zoogeográficas e totalizam cerca de 5400 espécies e 598 gêneros (Gagné, 2004). A fauna dos Cecidomyiidae neotropicais ainda é pouco conhecida e inclui cerca de 500 espécies em 170 gêneros.

Os Cecidomyiidae surgiram no Cretáceo e diversificaram-se intensamente com o surgimento das Angiospermae (Gagné, 1989). São usualmente divididos em quatro subfamílias: Catotrichinae, Lestremiinae, Porricondylinae e Cecidomyiinae. As três primeiras são pouco diversificadas e incluem essencialmente espécies fungívoras. Já os Cecidomyiinae têm hábitos variados e grande riqueza de espécies. Contêm formas fungívoras, mas principalmente fitófagas (galhadoras ou de vida livre) e predadoras.

\section{MORFOLOGIA E BIOLOGIA}

Os adultos (Fig. 1) são pequenos, variando de 1,0 a 5,0 mm de comprimento. São holópticos ou quase em ambos os sexos; antenas longas, flagelômeros geralmente com circunfilas e aparelho bucal reduzido. As asas são geralmente delicadas e com nervação reduzida em número, sendo a Costa geralmente contínua em torno da asa, usualmente com uma fratura na junção com R5 e mais fraca na margem posterior; R5 simples; M fraca ou ausente (exceto nos Lestremiinae); pernas geralmente longas e finas, esporão tibial ausente; tarsos com 5 artículos. As larvas são muito características (Fig. 2): têm a cápsula cefálica bastante reduzida e cônica, mandíbulas estiletiformes e diminutas; protórax geralmente com uma espátula esternal esclerotizada. O tegumento possui papilas variadas importantes para a sistemática. A pupa é adéctica e geralmente exarata (Fig. 3). Possui estruturas pontiagudas e esclerotizadas que a auxiliam a sair do solo, casulo ou da galha, tais como os chifres antenais e os espinhos faciais e abdominais.

Os adultos dos Cecidomyiidae têm vida efêmera e raramente se alimentam. A maioria reproduz-se sexuadamente, mas em alguns predadores e inquilinos é comum ocorrer pedogênese partenogenética (Gagné, 1989). A maioria das larvas é galhadora e muitas espécies são consideradas importantes pragas agrícolas, especialmente nas regiões Neártica e Paleártica (Barnes, 1946 a, b; 1948 a,b; 1949; 1951; 1956; Nijveldt, 1969; Fernandes, 1987).

\section{CECIDOMYIIDAE DA REGIÃO NEOTROPICAL}

Nos últimos 10 anos, muitas espécies e gêneros novos neotropicais foram descritos, tendo sido inclusive erigidas duas novas tribos para englobar parte dos Cecidomyiidae desta região; algumas espécies foram transferidas de gêneros e outras foram consideradas sinônimas. Parte desses resultados deve-se à revisão da fauna neotropical realizada por Gagné (1994), onde uma sinopse dos Cecidomyiidae neotropicais é apresentada e todos os gêneros e espécies conhecidos desta região, até aquele momento são listados. No entanto, o volume de informações produzidas desde então torna necessária uma atualização dos dados.

Os Cecidomyiidae apresentam cerca de 170 gêneros na Região Neotropical, sendo vários monotípicos e muitos conhecidos apenas por uma fase do ciclo evolutivo. De algumas espécies galhadoras desconhece-se a planta hospedeira, o que dificulta novas coletas. O material-tipo de diversas espécies está presumivelmente perdido, uma vez que não se encontra nas coleções indicadas como local de depósito. O conhecimento das subfamílias Lestremiinae e Porricondylinae é muito escasso, estando registrados apenas onze e oito gêneros, respectivamente, nesta região. Estas subfamílias são pouco diversificadas e como incluem apenas formas fungívoras e/ou fitófagas de vida livre não têm despertado o mesmo interesse que as formas galhadoras, incluídas em Cecidomyiinae.

Para o Brasil, estão registradas 159 espécies (de 75 gêneros). Deste montante, 95 espécies de 47 gêneros ocorrem no Estado do Rio de Janeiro, o que corresponde a 60\% do total. Além das espécies formalmente descritas, a fauna brasileira inclui ainda, cerca de 250 espécies não determinadas de Cecidomyiidae, cujas galhas foram descritas em revistas científicas e cujas plantas hospedeiras foram identificadas.

\section{ESTUDO TAXONÔMICO DOS CECIDOMYIIDAE NO BRASIL}

A fauna brasileira foi estudada de forma pioneira e intensa no início do século XX pelo jesuíta português J. S. Tavares. De 1906 a 1925, ele produziu uma vasta obra sobre os Cecidomyiidae do Brasil, descrevendo 26 gêneros e 56 espécies. Tavares é até hoje o grande nome da cecidologia brasileira. Infelizmente, grande parte do material obtido por este pesquisador está presumivelmente perdido. Mas, recentemente, E. Möhn (Staatliches Museum für Naturkunde, Sttuttgart, Alemanha) descobriu no Instituto Nun’Alvres, Caldas de Saúde (Portugal) espécimens que aparentemente fazem parte da Coleção Tavares. Esse material encontra-se no Staatliches Museum für Naturkunde.

E. H. Rübsaamen (Alemanha) e E. P. Felt (Estados Unidos) também contribuíram para o conhecimento da cecidologia brasileira, porém de forma mais modesta. No período de 1905 a 1908, Rübsaamen estudou a fauna de Cecidomyiidae do Brasil e do Peru, descrevendo 17 gêneros e 22 espécies. E. Felt, embora tenha se dedicado mais intensamente ao estudo da fauna norte-americana, esporadicamente descrevia material do Brasil (Felt, 1915a, 1915b). A coleção de Rübsaamen é propriedade do Zoologisches Museum der Humboldt Universität, Berlin (Alemanha), mas atualmente encontra-se no Staatliches Museum für Naturkunde. Já a coleção de E. P. Felt encontra-se no United States National Museum (Washington, USA). 

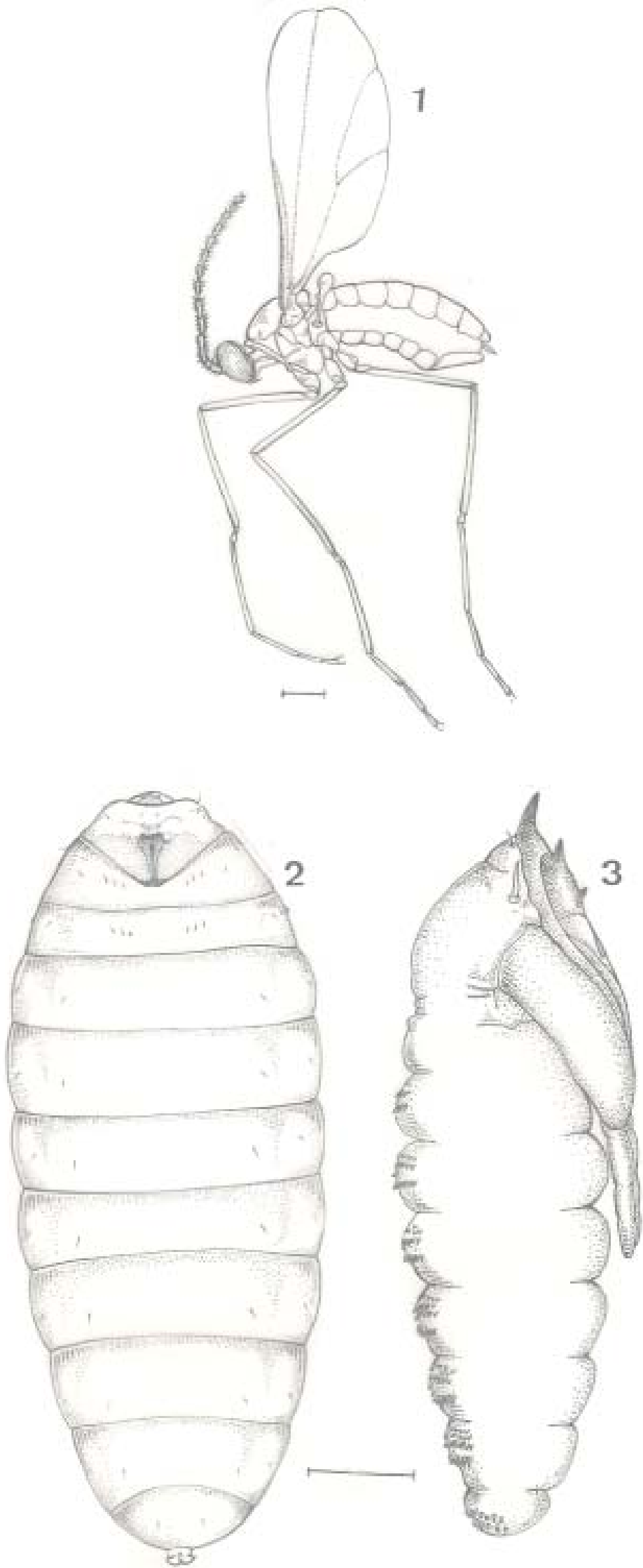
Desde Rübsaamen, trabalhos taxonômicos sobre a fauna brasileira foram realizados de forma esporádica por pesquisadores estrangeiros. Dentre estes, R. J. Gagné (Estados Unidos) é a principal referência, por seu extenso trabalho de revisão de toda a fauna neotropical, incluindo a brasileira (Gagné, 1994) e eventuais descrições de gêneros e espécies do Brasil.

A partir da década de 1990, o estudo da cecidologia brasileira ganhou novo impulso, devido à publicação de vários artigos tratando especificamente da fauna de restingas do Estado do Rio de Janeiro (Couri \& Maia, 1992; Maia, 1993a, b, c, d; 1994, 1995a, b, c; 1996a, b; 1999; 2001a, b; Maia \& Couri, 1992; Maia \& Monteiro, 1999; Maia 2002 in Madeira et al., 2002). Estes autores descreveram 10 gêneros e 47 espécies novas.

A grande quantidade de táxons descritos recentemente e a falta de dados sobre a diversidade deste grupo no Estado do Rio de Janeiro motivaram a realização deste catálogo, que ainda inclui novos registros de plantas hospedeiras e de espécies de cecidomiídeos. Embora os Cecidomyiidae estejam divididos em 4 subfamílias (Catotrichinae, Lestremiinae, Porricondylinae e Cecidomyiinae), apenas a última delas está representada, até o momento, no Estado do Rio, onde são encontrados exemplares de duas supertribos: Lasiopteridi e Cecidomyiidi. Neste catálogo, os Lasiopteridi são apresentados primeiro, em função de sua menor diversidade. Dentro das supertribos abordadas, adotou-se o critério de apresentar as espécies organizadas em tribos, e em gêneros, em ordem alfabética.

As seguintes siglas de museus foram utilizadas:

MNRJ: Museu Nacional/ Universidade Federal do Rio de Janeiro

SMNS: Staatliches Museum für Naturkunde, Sttuttgart, Alemanha

USNM: National Museum of Natural History, Washington, D. C., Estados Unidos da América

ZMHU: Zoologisches Museum der Humboldt Universität, Berlin, Alemanha

Cecidomyiidae do Rio de Janeiro

Subfamília Cecidomyiinae

Supertribo Lasiopteridi

Tribo Alycaulini

Gênero Alycaulus Rübsaamen

Alycaulus Rübsaamen, 1916a: 476. Espécie-tipo, mikaniae Rübsaamen (mon.)

globulus Gagné, 2001 (in Gagné et. al., 2001), figs. 9-
7. Holótipo macho, MNRJ, parátipos MNRJ e USNM. Localidade tipo: Brasil, RJ, Itatiaia, Rio de Janeiro, Silva Jardim. Planta hospedeira: Mikania glomerata Sprengel (Asteraceae). Distr. Brasil.

sp. (provavelmente espécie nova) Maia, 2001 a. Localidade: Brasil, RJ, Maricá e Arraial do Cabo. Planta hospedeira: Mikania hoehnei Robinson (Asteraceae). Distr.-Brasil.

\section{Gênero Baccharomyia Tavares}

Baccharomyia Tavares, 1917b: 129. Espécie-tipo, ramosina Tavares (des. orig.)

ramosina Tavares, 1917b: 130, fig. B (1-6). Síntipos: presumivelmente perdidos. Localidade tipo: Brasil, RJ, Nova Friburgo. Planta hospedeira: Baccharis trimera (Less.) DC. (Asteraceae). Distr. Brasil.

\section{Gênero Geraldesia Tavares}

Geraldesia Tavares, 1917b: 134. Espécie-tipo, eupatorii Tavares (des. orig.)

eupatorii Tavares, 1917b: 134, fig. C (1-5). Síntipos: presumivelmente perdidos. Localidade tipo: Brasil, RJ, Rio de Janeiro. Planta hospedeira: Eupatorium sp. (Asteraceae). Distr. Brasil, El Salvador.

\section{Gênero Neolasioptera Felt}

Neolasioptera Felt, 1908: 330. Espécie-tipo, Lasioptera vitinea Felt (des. subseq. Coquillet 1910: 575).

cerei Rübsaamen, 1905: 81 (Lasioptera). Síntipos: ZMHU. Localidade tipo: Brasil, RJ, Cabo Frio. Planta hospedeira: Selenicereus setaceus (SD) Berg. (Cactaceae). Distr. Brasil. Ref.: Maia (1999) (descrição do macho, pupa e larva de terceiro ínstar).

cupheae Gagné, 1998: 522, figs. 1-9 (in GaGnÉ, Ferraz \& Monteiro, 1998). Holótipo macho, MNRJ, parátipos MNRJ e USNM. Localidade tipo: Brasil, RJ, Silva Jardim. Planta hospedeira: Cuphea carthagenensis (Jacq.) (Lythraceae). Distr. Brasil.

eugeniae Maia, 1993a: 718. Holótipo macho, MNRJ, parátipos MNRJ. Localidade tipo: Brasil, RJ, Maricá. Outras localidades: Arraial do Cabo e Rio de Janeiro (Paquetá). Planta hospedeira: Eugenia uniflora L. (Myrtaceae). Distr. Brasil. Refs.: Maia (1995c); Maia \& Couri (1997).

sp.1. Maia, 2001 a. Localidade: Brasil, RJ, Maricá, Carapebus e Arraial do Cabo. Planta hospedeira: Arrabidaea conjugata (Vell.) Mart. (Bignoniaceae). Distr.-Brasil.

sp.2. Maia, 2001 a. Localidade: Brasil, RJ, Maricá. Planta hospedeira: Inga maritima Benth (Leguminosae). Distr.-Brasil. 


\section{Gênero Smilasioptera Möhn}

Smilasioptera Möhn, 1975: 10. Espécie-tipo, candelariae Möhn (des. orig.).

candelariae Möhn, 1975: 11, pl. 2, figs. 20-28, pl. 3, figs. 1-9. Holótipo macho, SMNS. Localidade-tipo: El Salvador, Santa Ana, SE Candelaria. Planta hospedeira: Smilax mexicana Griseb. ex Kunth (Smilacaceae). Distr.- Brasil e El Salvador.

Tribo Camptoneuromyiini

\section{Gênero Camptoneuromyia Felt}

Camptoneuromyia Felt, 1908: 334. Espécie-tipo, Dasineura virginica Felt (des. subseq. Coquillet 1910: 518).

sp. Maia, 2001a. Localidade: Brasil, RJ, Maricá. Planta hospedeira: Jacquemontia holosericea (Weinman) O’ Donell (Convolvulaceae). Distr.-Brasil.

\section{Gênero Meunieriella Kieffer}

Meunieria Rübsamen, 1905: 137. Espécie-tipo, dalechampiae Rübsamen (des. orig. como n. g., n. sp). Preocc. Kieffer, 1904.

Meunieriella Kieffer, 1909: 35, novo nome para Meunieria Rübsaamen. Espécie-tipo, Meunieria dalechampiae Rübsamen (aut.).

Dolicholabis Tavares, 1918b: 72. Espécie-tipo, lantanae Tavares (des. orig.).

dalechampiae Rübsaamen, 1905: 137. Síntipos: ZMHU. Localidade tipo: Brasil, RJ, Rio de Janeiro. Planta hospedeira: Dalechampia ficifolia Lam. (Euphorbiaceae). Distr. Brasil.

\section{Tribo Oligotrophini}

Gênero Apodiplosis Tavares

Apodiplosis Tavares, 1922: 37. Espécie-tipo, praecox Tavares (des. orig.).

praecox Tavares, 1922: 35, figs. 7,8. Síntipos: presumivelmente perdidos. Localidade tipo: Brasil, RJ, Nova Friburgo. Planta hospedeira: Psychotria sp. (Rubiaceae). Distr. Brasil.

\section{Gênero Calmonia Tavares}

Calmonia Tavares, 1917b: 173. Espécie-tipo, urostigmata Tavares (des. orig.).

urostigmata Tavares, 1917b: 173, pl. X, figs. 10-12, pl. XI, fig. 9. Síntipos: parte presumivelmente perdida; machos e fêmeas, possivelmente síntipos depositados na Coleção Felt. Localidade tipo: Brasil, RJ, Nova Friburgo. Planta hospedeira: Ficus sp. (Moraceae). Distr. Brasil.

\section{Gênero Dasineura Rondani}

Dasineura Rondani, 1840: 17. Espécie-tipo, obscura Rondani (des. subseq. Rondani 1856: 200).

Dasyneura Agassiz \& Loew, 1846: 11, emenda de Dasineura.

Perrisia Rondani 1846: 371. Espécie-tipo, Cecidomyiae urticae Perris (des. orig.)

copabanensis Maia, 1993a: 717, figs. 1-8. Holótipo macho, MNRJ, parátipos MNRJ. Localidade tipo: Brasil, RJ, Arraial do Cabo. Planta hospedeira: Eugenia copacabanensis Kiaersk. (Myrtaceae). Distr. Brasil.

couepiae Maia, 2001 b. Holótipo, macho, MNRJ, parátipos MNRJ. Localidade tipo: Brasil, RJ, Maricá. Outras localidades: RJ: Arraial do Cabo. Planta hospedeira: Couepia ovalifolia (Schott) Benth. (Chrysobalanaceae). Distr. Brasil.

globosa Maia, 1995b: 1002, figs. 1-10. Holótipo macho, MNRJ. Parátipos MNRJ Localidade tipo: Brasil, RJ, Maricá. Outras localidades: RJ: Arraial do Cabo, Niterói. Planta hospedeira: Eugenia rotundifolia Casar. (Myrtaceae). Distr. Brasil. Refs.: Maia (1995b); Maia \& Couri, (1997); Maia (1999).

myrciariae Maia, 1995b: 1003, figs. 11-22. Holótipo, macho, MNRJ, parátipos MNRJ Localidade tipo: Brasil, RJ, Maricá. Planta hospedeira: Myrciaria floribunda (West ex Willdenow) Berg. (Myrtaceae). Distr. Brasil.Refs.: Maia (1995b); Maia \& Couri (1997).

tavaresi Maia, 1995b: 1006, figs. 23-33. Holótipo, fêmea, MNRJ. Parátipos, MNRJ Localidade tipo: Brasil, RJ, Maricá. Planta hospedeira: Neomitranthes obscura (DC.) N. J. E. Silveira (Myrtaceae). Distr. Brasil. Refs.: Maia (1995b); Maia \& Couri (1997).

\section{Gênero Mayteniella Maia}

Mayteniella Maia, 2001b. Espécie-tipo, distincta Maia (des. orig.).

distincta Maia, 2001 b, figs. 89-96. Holótipo macho, MNRJ, parátipos, MNRJ. Localidade tipo: Brasil, RJ, Maricá. Outras localidades: RJ: Arraial do Cabo, Carapebus e Rio de Janeiro (Paquetá). Planta hospedeira: Maytenus obtusifolia Mart. var. obovata Mart. (Celastraceae). Distr. Brasil. Ref.: Pamplona, et al. (2000).

\section{Gênero Myrciariamyia Maia}

Myrciariamyia Maia, 1994: 635, figs. 1, 4-6, 8-11, 13. Espécie-tipo, bivalva Maia (des. orig.).

bivalva Maia, 1994: 637, figs. 2-5, 7-8, 10-17. Holótipo macho, MNRJ, parátipos, MNRJ. Localidade tipo: Brasil, RJ, Maricá. Planta hospedeira: Myrciaria floribunda (West ex Willdenow) Berg. (Myrtaceae). Distr. Brasil. Ref.: Maia(1995b). 


\section{Gênero Neomitranthella Maia}

Neomitranthella Maia, 1995a: 571, figs. 15, 18-22. Espécie-tipo, robusta Maia (des. orig.).

robusta Maia, 1995a: 571, figs. 16-18, 20-28. Holótipo macho, MNRJ. Parátipos, MNRJ. Localidade tipo: Brasil, RJ, Maricá. Outras localidades: RJ: Arraial do Cabo e Carapebus. Planta hospedeira: Neomitranthes obscura (DC.) N. J. E. Silveira (Myrtaceae). Distr. Brasil. Refs.: Maia (1995b); Maia \& Couri (1997).

\section{Gênero Paulliniamyia Maia}

Paulliniamyia Maia, 2001 b. Espécie-tipo, ampla Maia (des. orig.).

ampla Maia, 2001 b, figs. 106-113. Holótipo macho, MNRJ, parátipos, MNRJ. Localidade tipo: Brasil, RJ, Maricá. Outras localidades: RJ: Arraial do Cabo e Carapebus. Planta hospedeira: Paullinia weinmanniaefolia Mart. (Sapindaceae). Distr. Brasil.

\section{Tribo Trotterini}

Gênero Trotteria Kieffer, 1901

Trotteria Kieffer, 1901: 561, novo nome para Choristoneura Rübsaamen. Espécie-tipo, Cecidomyia obtusa Loew (aut.)

Choristoneura Rübsaamen, 1892: 342. Espécie-tipo, Cecidomyia obtusa Loew (mon.). preoc. Ledere 1859.

quadridentata Maia, 2001 c. Localidade: Brasil, RJ, Maricá. Planta hospedeira: Pouteria caimito (R. \& P.) Radlk. var. laurifolia (Gomes) Baehni (Sapotaceae). Distr.-Brasil.

sp. 1. Maia, 1995b: 1010. Localidade: Brasil, RJ, Maricá. Planta hospedeira: Eugenia copacabanensis Kiaersk. (Myrtaceae). Distr.-Brasil. Ref.: Maia (1995c).

\section{Supertribo Cecidomyiidi}

Tribo Anadiplosini

Gênero Anadiplosis Tavares

Anadiplosis Tavares, 1916: 41. Espécie-tipo, pulchra Tavares (des. orig. )

pulchra Tavares, 1916: 42, figs. 3-6. Síntipos: parte presumivelmente perdida, parte depositada na Coleção Felt. Localidade tipo: Brasil, RJ, Nova Friburgo. Planta hospedeira: Machaerium sp. (Fabaceae). Distr.-Brasil. Ref.: Tavares (1920c).

venusta Tavares, 1916: 46, figs. 7 (A-E) - 9. Síntipos: presumivelmente perdidos. Localidade-tipo: Brasil, RJ, Nova Friburgo. Planta hospedeira: Machaerium sp. (Fabaceae). Distr.-Brasil. Ref.: Tavares (1920b, c).

\section{Gênero Uleella Rübsaamen}

Uleella Rübsaamen, 1907: 120. Espécie-tipo, dalbergiae Rübsaamen (mon.).

Uleela Gagné, 1968: 26, error.

dalbergiae Rübsaamen, 1907: 121. Síntipos ZMHU. Localidade tipo: Brasil, RJ, Jacarepaguá. Planta hospedeira: Dalbergia sp. (Leguminosae). Distr.-Brasil.

\section{Tribo Asphondyliini}

Subtribo Asphondyliina

Gênero Asphondylia Loew, 1850: 21, 37. Espécie-tipo, Cecidomyia sarothammi Loew (des. subs. Karsch 1877: 15).

Gisonobasis Rübsaamen 1916 a: 432. Espécie-tipo, tournefortiae Rübsaamen (des. subs.Rübsaamen 1916 b: 12).

Eoasphondylia (como subgênero de Asphondylia) Möhn, 1960: 214. Espécie-tipo, Asphondylia convolvuli Möhn (des. orig.)

borreriae Rübsaamen, 1905: 74. Síntipos, ZMHU ? Localidade tipo: Brasil, RJ, Harpoador. Outras localidades: RJ: Arraial do Cabo, Maricá e Carapebus. Planta hospedeira: Borreria sp. e Diodia sp. (Rubiaceae). Distr.-Brasil. Refs.: Rübsamen (1907); Maia, Couri \& Monteiro (1992) (descrição da larva, macho e fêmea).

communis Maia \& Couri, 1992: 658, figs. 25-27 (in Maia, Couri \& Monteiro, 1992). Holótipo macho, MNRJ, parátipos, MNRJ. Localidade tipo: Brasil, RJ, Maricá. Planta hospedeira: Ximenia americana L. var. americana (Olacaceae). Distr.-Brasil. Ref.: Maia (1999) (descrição da larva, macho e fêmea).

cordiae Möhn, 1959: 338, pl. 40, figs. 75-77. Holótipo macho, SMNS, parátipos, SMNS. Localidade tipo: El Salvador, La Libertad, W Ateos. Planta hospedeira: Cordia alba (Jacq.) Roem. \& Schult., C. dentata Poir e C. verbenacea DC. (Boraginaceae). Distr.-Brasil (RJ: Maricá e Carapebus) e El Salvador. Ref.: Maia (2001a).

glomeratae Gagné, 2001 (in Gagné, Oda \& Monteiro, 2001): figs.: 18-22, 28-29. Holótipo macho, MNRJ, parátipos MNRJ. Localidade tipo: Brasil, RJ, Rio de Janeiro, Parati, Teresópolis e Silva jardim (Reserva Biológica de Poço das Antas). Planta hospedeira: Mikania glomerata Sprengel (Asteraceae). Distr.-Brasil.

maricensis Maia \& Couri, 1992: 658, figs. 25-27 (in Maia et al., 1992). Holótipo fêmea, MNRJ, parátipos, MNRJ. Localidade tipo: Brasil, RJ, Maricá. Planta hospedeira: Struthanthus maricensis Rizz. (Loranthaceae). Distr.-Brasil. moehnei Skuhravá, 1989: 203. Holótipo macho, Coleção Tavares. Planta hospedeira: Mikania guaco Humb. \& Bonpl. (Asteraceae). Distr.-Brasil. Refs.: Gagné et al. (2001). 
tavaresi Möhn, 1973: 4, pl. 1, figs. 7-13 (Asphondylia), preoc. por Rübsaamen, 1916. Holótipo macho, Coleção Tavares. Localidade-tipo: Brasil, Rio Grande do Sul, São Leopoldo.

peploniae Maia, 2001 b: figs. 8-14. Holótipo macho, MNRJ, parátipos, MNRJ. Localidade tipo: Brasil, RJ, Carapebus. Planta hospedeira: Peplonia asteria (Vell.) Font. \& Schw. (Asclepiadaceae). Distr.-Brasil.

sennae Maia \& Couri, 1992: 656, fig. 17 (in Maia et al., 1992). Holótipo fêmea, MNRJ, parátipos, MNRJ. Localidade tipo: Brasil, RJ, Maricá. Planta hospedeira: Senna bicapsularis (L.) Roxb. (Leguminosae). Distr.-Brasil.

ulei Rübsaamen, 1907: 172. Síntipos, ZMHU ? Localidade tipo: Brasil, RJ, Palmeiras. Planta hospedeira: Mikania sp. (Asteraceae). Distr.-Brasil. Ref.: Möhn (1973).

sp.1. Maia, 2001 a. Localidade: Brasil, RJ, Carapebus. Planta hospedeira: Vernonia rufogrisea St. Hill (Asteraceae). Distr.-Brasil.

sp.2. Maia, 2001 a. Localidade: Brasil, RJ, Carapebus e Maricá. Planta hospedeira: Erythroxylum ovalifolium Peyr. Distr.-Brasil.

sp.3. Pamplona et al., 2000. Localidade: Brasil, RJ, Rio de Janeiro (Paquetá). Planta hospedeira: Porophyllum ruderale (Jacq.) Cass. (Asteraceae). Distr.-Brasil.

\section{Gênero Bruggmanniella Tavares}

Bruggmanniella Tavares, 1909: 19. Espécie-tipo, braziliensis Tavares (mon.).

Hemibruggmanniella Möhn, 1961: 6. Espécie-tipo, Bruggmanniella oblita Tavares (des. orig.).

byrsonimae Maia \& Couri, 1992: 657, figs. 18-24 (Asphondylia). Holótipo macho, MNRJ, parátipos, MNRJ. Localidade tipo: Brasil, RJ, Maricá. Outras localidades: RJ, Carapebus. Planta hospedeira: Byrsonima sericea DC. (Malpighiaceae) Distr.-Brasil. Ref.: Maia (1999).

maytenuse Maia \& Couri, 1992: 655, figs. 12-16 (Asphondylia). Holótipo fêmea, MNRJ, parátipos, MNRJ. Localidade tipo: Brasil, RJ, Maricá. Planta hospedeira: Maytenus obtusifolia Mart. var. obovata Mart. (Celastraceae). Distr.-Brasil. Ref.: Maia (1999) (descrição da larva).

oblita Tavares, 1920a: 34. Lectótipo, fêmea, Coleção Tavares. Localidade tipo: Brasil, RJ, Nova Friburgo. Planta hospedeira: Schinnus sp. (Anacardiaceae). Distr.-Brasil. Ref.: Möhn (1961: 7) (redescr. larva, pupa e fêmea).

\section{Gênero Parazalepidota Maia}

Parazalepidota Maia, 2001 b. Espécie-tipo, clusiae Maia (des. orig.)

clusiae Maia, 2001 b: figs. 5-107. ). Holótipo macho, MNRJ, parátipos, MNRJ. Localidade tipo: Brasil,
RJ, Maricá. Outras localidades: RJ, Arraial do Cabo e Niterói. Planta hospedeira: Clusia fluminensis Tr. \& Pl. (Clusiaceae). Distr.-Brasil.

Gênero Perasphondylia Möhn

Perasphondylia Möhn, 1960: 218. Espécie-tipo, reticulata Möhn (des. orig.).

mikaniae Gagné, 2001 (in Gagné et al., 2001): figs.: 30-31, 70-75. Holótipo macho, MNRJ, parátipos MNRJ. Localidade tipo: Brasil, RJ, Silva Jardim (Reserva Biológica de Poço das Antas), Parati e Parque Nacional de Itatiaia). Planta hospedeira: Mikania glomerata Sprengel (Asteraceae).

\section{Gênero Rhoasphondylia Möhn}

Rhoasphondylia Möhn, 1960: 224. Espécie-tipo, Oxasphondylia friburgensis Tavares (des. orig.).

friburgensis Tavares, 1917b: 126, fig. A(1-6). Síntipos: presumivelmente perdidos. Localidade-tipo: Brasil, RJ, Nova Friburgo. Planta hospedeira: Baccharis dracunculifolia DC., B. schultzii Baker e B. trinervis Pers. (Asteraceae). Distr.-Brasil. Ref.: Möhn (1960).

\section{Gênero Zalepidota Rübsaamen}

Zalepidota Rübsaamen, 1907: 38. Espécie-tipo, piperis Rübsaamen (mon.).

Ozobia Kieffer, 1913: 97. Espécie-tipo, tavaresi Kieffer (des. orig.)

Oxasphondylia Felt, 1915b: 204. Espécie-tipo, reticulata Felt (des. orig.).

piperis Rübsamen 1907: 121. Síntipos: ZMHU ? Localidade-tipo: Brasil, RJ, Rio de Janeiro (Tijuca). Planta hospedeira: Piper sp. (Piperaceae). Distr.-Brasil. Refs.: Rübsaamen (1908) e Möhn (1963).

\section{Subtribo Schizomyiina}

\section{Gênero Anasphondylia Tavares}

Anasphondylia Tavares, 1920a: 38. Espécie-tipo, Asphondylia myrtacea Tavares (des. orig.).

myrtaceae Tavares, 1920b: 125, pl. III, figs. 10-13. Síntipos: presumivelmente perdidos. Localidade-tipo: Brasil, RJ, Nova Friburgo. Planta hospedeira: espécie indeterminada de Myrtaceae. Distr.-Brasil. Ref.: Tavares (1922).

\section{Gênero Bruggmannia Tavares}

Bruggmannia Tavares, 1906: 81. Espécie-tipo, braziliensis Tavares (des. orig.).

Feltomyia Kieffer 1913: 100. Espécie-tipo, Uleella pisonifolia Felt (des. orig.). 
acaudata Maia, 2004: 761, figs. 1-19. Holótipo macho, MNRJ, parátipos, MNRJ. Localidade-tipo: Brasil, RJ, Maricá. Outras localidades: RJ, Arraial do Cabo, Maricá, Carapebus e Rio de Janeiro (Paquetá). Planta hospedeira: Guapira opposita (Vell.) Reitz. (Nyctaginaceae). Distr.-Brasil. Ref.: Maia (2001a).

elongata Maia \& Couri, 1992: 210, figs. 1-14. Holótipo macho, MNRJ, parátipos, MNRJ. Localidade-tipo: Brasil, RJ, Maricá. Outras localidades: RJ, Arraial do Cabo, Carapebus e Maricá. Planta hospedeira: Guapira opposita (Vell.) Reitz. (Nyctaginaceae). Distr.-Brasil. Ref.: Maia \& Monteiro (1999).

lignicola Kieffer, 1913: 101 (sem descrição, cita Rübsaamen, 1905: 133) (Uleella). Síntipo: ZMHU ?. Localidade-tipo: Brasil, RJ, Serra do Macahé. Planta hospedeira: Neea sp. (Nyctaginaceae) (orig. como Dalbergia sp.). Distr.-Brasil.

longicauda Kieffer, 1913: 101 (sem descrição, cita Rübsaamen, 1908: 26) (Uleella). Síntipo: ZMHU ?. Localidade-tipo: Brasil, RJ, Rio de Janeiro. Planta hospedeira: Neea sp. (Nyctaginaceae). Distr.-Brasil.

monteiroi Maia \& Couri, 1992: 214, figs. 26-31. Holótipo fêmea, MNRJ, parátipos, MNRJ Localidade-tipo: Brasil, RJ, Maricá. Planta hospedeira: Guapira opposita (Vell.) Reitz. (Nyctaginaceae). Distr.-Brasil.

neeana Kieffer, 1913: 101 (sem descrição, cita Rübsaamen, 1907: 121, fig. 3) (Uleella). Síntipo: ZMHU ?. Localidade-tipo: Brasil, RJ, Rio de Janeiro. Planta hospedeira: Neea sp. (Nyctaginaceae). Distr.-Brasil.

robusta Maia \& Couri, 1992: 212, figs. 15-25. Holótipo macho, MNRJ, parátipos, MNRJ. Localidade-tipo: Brasil, RJ, Maricá. Outras localidades: RJ, Arraial do Cabo, Carapebus e Paquetá. Planta hospedeira: Guapira opposita (Vell.) Reitz. (Nyctaginaceae). Distr.-Brasil. Ref.: Maia \& Monteiro (1999).

\section{Gênero Pisphondylia Möhn, 1964}

Pisphondylia Möhn, 1964: 344. Espécie-tipo, salvadorensis Möhn (des. orig.)

brasiliensis Couri \& Maia, 1992: 730, figs. 1-2. Holótipo macho, MNRJ, parátipos, MNRJ. Localidade-tipo: Brasil, RJ, Maricá. Planta hospedeira: Guapira opposita (Vell.) Reitz. (Nyctaginaceae). Distr.-Brasil.

\section{Gênero Proasphondylia Felt}

Proasphondylia Felt, 1915b: 203. Espécie-tipo, brasiliensis (des. orig.)

formosa Maia, 1993b: 217, figs.18-11. Holótipo macho, MNRJ, parátipos, MNRJ. Localidade-tipo: Brasil, RJ, Maricá. Planta hospedeira: Guapira opposita (Vell.) Reitz. (Nyctaginaceae). Distr.-Brasil.

guapirae Maia, 1993b: 216, figs.1-6. Holótipo macho, MNRJ, parátipos, MNRJ. Localidade-tipo: Brasil, RJ, Arraial do Cabo. Planta hospedeira: Guapira opposita (Vell.) Reitz. (Nyctaginaceae). Distr.-Brasil. Ref.: Maia (1999) (descrição da larva).

\section{Gênero Schizomyia Kieffer}

Schizomyia Kieffer, 1889: 183. Espécie-tipo, galiorum Kieffer (mon.)

sp.1. Maia, 2001 a. Localidade: Brasil, RJ, Maricá. Planta hospedeira: Jacquemontia holosericea (Weinman) O’Donell (Convolvulaceae). Distr.-Brasil.

sp.2. Maia, 2001 a. Localidade: Brasil, RJ, Maricá. Planta hospedeira: Sebastiania glandulosa (Mart.) Pax. (Euphorbiaceae). Distr.-Brasil.

sp.3. Localidade: Brasil, RJ, Rio de Janeiro (Grumari). Planta hospedeira: Stachyrtapheta sp. (Verbenaceae). Distr.Brasil.

sp.4. Gagné, 1994. Localidade: Brasil, RJ. Planta hospedeira: Psittacanthus robustus (Martius in Schultes \& J. H. Schultes) Mart. (Loranthaceae). Distr.-Brasil.

\section{Gênero Stephomyia Tavares}

Stephomyia Tavares, 1916: 54. Espécie-tipo, eugeniae Tavares (mon.)

\section{epeugeniae Gagné, 1994: 144.}

eugeniae Tavares, 1916: 54, figs. 12-14. Homônimo secundário de eugeniae Felt. Lectótipo (Möhn, 1962: 234) na Coleção Tavares; parte da série original na Coleção Felt. Localidade-tipo: Brasil, RJ, Rio de Janeiro (Jardim Botânico). Planta hospedeira: Eugenia sp. (Myrtaceae). Distr.-Brasil.

espiralis Maia, 1993c: 526, figs. 16-20. Holótipo fêmea, MNRJ, parátipos, MNRJ. Localidade-tipo: Brasil, RJ, Arraial do Cabo e Maricá. Planta hospedeira: Eugenia copacabanensis Kiaersck (Myrtaceae). Distr.-Brasil. Refs.: Maia (1995c); Maia \& Couri (1997); Maia (1999) (descrição da pupa).

mina Maia, 1993c: 528, figs. 21-23. Holótipo macho, MNRJ, parátipos, MNRJ. Localidade-tipo: Brasil, RJ, Maricá. Outras localidades: RJ, Carapebus. Planta hospedeira: Neomitranthes obscura (DC) N. J. E. Silveira (Myrtaceae). Distr.-Brasil. Refs.: Maia (1995c); Maia \& Couri (1997).

rotundifoliorum Maia, 1993c: 523, figs. 1-5. Holótipo macho, MNRJ, parátipos, MNRJ. Localidade-tipo: Brasil, RJ, Maricá. Outras localidades: RJ, Arraial do Cabo. Planta hospedeira: Eugenia rotundifolia Casar (Myrtaceae). Distr.Brasil. Refs.: Maia (1995c); Maia \& Couri (1997); Maia (1999).

tetralobae Maia, 1993c: 525, figs. 10-15. Holótipo fêmea, MNRJ, parátipos, MNRJ. Localidade-tipo: Brasil, RJ, Maricá. Planta hospedeira: Eugenia copacabanensis Kiaersck (Myrtaceae). Distr.-Brasil. Refs.: Maia (1995c); Maia \& Couri (1997). 
sp. Maia, 2001a. Localidade: Brasil, RJ, Carapebus. Planta hospedeira: Eugenia multiflora Camb. (Myrtaceae). Distr.-Brasil.

\section{Tribo Cecidomyiini}

\section{Gênero Contarinia Rondani}

Contarinia Rondani 1860: 289. Espécie-tipo, Tipula loti DeGeer (des. orig.).

gemmae Maia, 2002 (in Madeira et al.,2002): 45, figs. 68-83b. Holótipo macho, MNRJ, parátipos MNRJ. Localidade-tipo: Brasil, RJ, Carapebus. Planta hospedeira: Calophyllum brasiliense Camb. (Clusiaceae). Distr.-Brasil.

ubiquita Gagné, 2001 (in Gagné et al., 2001): figs.: 3233. Holótipo larva, MNRJ, parátipos MNRJ. Localidade-tipo: Brasil, RJ, Silva Jardim (Reserva Biológica de Poço das Antas), Parque Nacional da Serra dos Órgãos e Parati. Planta hospedeira: Mikania glomerata Sprengel (Asteraceae). Em galhas de Perasphondylia mikaniae Gagné, 2001 (in Gagné et al., 2001).

sp.1. Maia, 2001 a. Localidade: Brasil, RJ, Maricá. Planta hospedeira: Manilkara subsericea (Mart.) Dubard (Sapotaceae). Distr.-Brasil.

sp.2. Maia, 2001 a. Localidade: : Brasil, RJ, Maricá. Planta hospedeira: Ouratea cuspidata (St. Hill) Engl. (Ochnaceae). Distr.-Brasil.

sp.3. Gagné, 1994. Localidade: Brasil, RJ. Planta hospedeira: Heteropteris sp. (Malpighiaceae). Distr.-Brasil.

\section{Tribo Clinodiplosini}

\section{Gênero Cleitodiplosis Tavares}

Cleitodiplosis Tavares, 1921: 108. Type species, Necrophlebia graminis Tavares (mon.).

graminis Tavares, 1916: 37, figs. 1 A-C, 2 (Necrophlebia). Síntipos: parte presumivelmente perdida e parte na Coleção Felt. Localidade-tipo: Brasil, RJ. Planta hospedeira: Paspalum conjugatum Bergius (Poaceae). Distr.-Brasil.

\section{Gênero Clinodiplosis Kieffer}

Clinodiplosis Kieffer, 1895: cclxxx. Espécie-tipo, Diplosis cilicrus Kieffer (des. orig.).

chlorophora Rübsaamen, 1905: 82. Síntipos, ZMHU. Localidade-tipo: Brasil, RJ, Fabrica. Planta hospedeira: Chlorophora tinctoria (L.) Gaudich. ex Benth. (Moraceae). Distr.-Brasil.

diodiae Maia, 2001b, figs. 15-21. Holótipo macho, MNRJ, parátipos, MNRJ. Localidade-tipo: Brasil, RJ, Carapebus. Planta hospedeira: Diodia gymnocephala (DC.) K. Schum. (Rubiaceae). Distr.-Brasil. marcetia Tavares, 1917a: 22, figs. 1-5 (Eudiplosis). Síntipos: parte presumivelmente perdida e parte na Coleção Felt. Localidade-tipo: Brasil, RJ, Nova Friburgo. Planta hospedeira: Marcetia sp. (Melastomataceae). Distr.-Brasil.

melissae Maia, 1993d: 695, figs. 1-12. Holótipo macho, MNRJ, parátipos, MNRJ. Localidade-tipo: Brasil, RJ, Maricá. Planta hospedeira: Melissa officinalis L. (Labiatae). Distr.-Brasil.

profusa Maia, 2001b, figs. 22-30. Holótipo macho, MNRJ, parátipos, MNRJ. Localidade-tipo: Brasil, RJ, Maricá. Outras localidades: RJ, Arraial do Cabo. Planta hospedeira: Eugenia uniflora L. (Myrtaceae). Distr.-Brasil.

rubiae Tavares 1918a: 70, pl. III, fig. 12 (Eudiplosis). Síntipos: presumivelmente perdida. Localidade-tipo: Brasil, RJ, Nova Friburgo. Planta hospedeira: Rubia sp. (Rubiaceae). Distr.-Brasil.

sp.1. Maia, 2001a. Localidade: Brasil, RJ, Maricá. Planta hospedeira: Atronium sp. (Anacardiaceae). Distr.-Brasil.

sp.2. Maia, 2001a. Localidade: Brasil, RJ, Carapebus. Planta hospedeira: Peplonia asteria (Vell.) Font. \& Schw. (Asclepiadaceae). Distr.-Brasil.

sp.3. Maia, 2001a. Localidade: Brasil, RJ, Maricá. Planta hospedeira: Mikania hoehnei Robinson (Asteraceae). Distr.-Brasil.

sp.4. Maia, 2001a. Localidade: Brasil, RJ, Maricá e Carapebus. Planta hospedeira: Erythroxyllum ovalifolium Peyr. (Erythroxylaceae). Distr.-Brasil.

sp.5. Maia, 2001a. Localidade: Brasil, RJ, Maricá. Planta hospedeira: Heteropteris nitida DC. (Malpighiaceae). Distr.-Brasil.

sp.6. Maia, 2001a. Localidade: Brasil, RJ, Maricá e Carapebus. Planta hospedeira: Neomitranthes obscura (DC) N. J. E. Silveira (Myrtaceae). Distr.-Brasil.

sp.7. Maia, 2001a. Localidade: Brasil, RJ, Carapebus. Planta hospedeira: Passiflora mucronata Lam. (Passifloraceae). Distr.-Brasil.

sp.8. Maia, 2001a. Localidade: Brasil, RJ, Maricá e Carapebus. Planta hospedeira: Paullinia weinmanniaefolia Mart. (Sapindaceae). Distr.-Brasil.

sp.9. Maia, 2001a. Localidade: Brasil, RJ, Maricá e Carapebus. Planta hospedeira: Smilax rufescens Griseb. (Smilacaceae). Distr.-Brasil.

sp.10. Rübsaamen, 1907. Brasil, RJ. Planta hospedeira: Mikania guaco Bonpl. e Mikania sp. (Asteraceae). Distr.-Brasil.

Gênero Iatrophobia Rübsaamen

Iatrophobia Rübsaamen, 1916a: 469. Espécie-tipo, Clinodiplosis brasiliensis Rübsaamen (mon.).

Jatrophobia Barnes 1946: 32. Emend. brasiliensis Rübsaamen, 1907: 156 (Clinodiplosis). 
Síntipos, ZMHU. Localidade-tipo: Brasil, RJ e Santa Catarina, Tubarão. Planta hospedeira: Manihot dichotoma Ule, M. palmata Müll. Arg. e M. utilissima Pohl. (Euphorbiaceae). Distr.-Brasil, Colombia, Costa Rica, Guiana, Peru, St. Vincent, Tobago e Trinidad. Refs.: Rübsaamen (1916a), Tavares (1918a), Korytkowski \& Sarmiento (1967) (como Hyperdiplosis sp.).

\section{Gênero Liodiplosis Gagné}

Liodiplosis Gagné, 2001 (in Gagné et al., 2001). Espécie-tipo, cylindrica Gagné, 2001 (in Gagné et al., 2001) (des. orig.)

conica Gagné, 2001 (in Gagné, Oda \& Monteiro, 2001): figs. 43, 47, 51-52, 56. Holótipo macho, MNRJ, parátipos, MNRJ. Localidade-tipo: Brasil, RJ, Rio de Janeiro (Parque Nacional da Tijuca) e Silva Jardim (Reserva Biológica de Poço das Antas). Planta hospedeira: Mikania glomerata Sprengel (Asteraceae). Distr.-Brasil.

cylindrica Gagné, 2001 (in Gagné et al., 2001): figs.: 35-42, 46, 49-50, 55. Holótipo macho, MNRJ, parátipos, MNRJ. Localidade-tipo: Brasil, RJ, Silva Jardim (Reserva Biológica de Poço das Antas). Planta hospedeira: Mikania glomerata Sprengel (Asteraceae). Distr.-Brasil.

spherica Gagné, 2001 (in Gagné et al., 2001): figs. 44, 48, 53-54, 57-58. Holótipo macho, MNRJ, parátipos, MNRJ. Localidade-tipo: Brasil, RJ, Parati e Silva Jardim (reserva Biológica de Poço das Antas). Planta hospedeira: Mikania glomerata Sprengel (Asteraceae). Distr.-Brasil.

\section{Gênero Schismatodiplosis Rübsaamen}

Schismatodiplosis Rübsaamen, 1916a: 467. Espécietipo, Clinodiplosis lantanae Rübsaamen (mon.).

lantanae Rübsaamen, 1907: 151 (Clinodiplosis). Síntipos: ZMHU. Localidade-tipo: Brasil, RJ (Cabo Frio e Palmeiras) e Santa Catarina (Tubarão). Outras localidades: RJ, Paquetá e Niterói. Planta hospedeira: Lantana camara L., L. hispida Kunth, L. urticifolia e Lantana spp. (Verbenaceae). Distr. - Brasil, México e Trinidad. Refs.: Rübsaamen (1916a), Tavares (1917a) e Pamplona et al. (2000).

\section{Tribo Lestodiplosini}

\section{Gênero Lestodiplosis Kieffer}

Leptodiplosis Kieffer, 1894a: xxviii. Grafia original invalidada (Comissão Internacional de Nomenclatura Zoológica 1958).

Lestodiplosis Kieffer, 1894b: 84. Espécie-tipo, Cecidomyia pictipennis Perris (des. subseq. Kieffer, 1895 a: cclxxx).

Coprodiplosis Kieffer 1894b: 84. Espécie-tipo, cryphali Kieffer (mon.). braziliensis Tavares, 1920b: 113 (Coprodiplosis). Síntipos: presumivelmente perdidos. Localidade-tipo: Brasil, RJ, Nova Friburgo. Hospedeiro: ex galha de Anadiplosis pulchra Tavares, 1916 (Diptera, Cecidomyiidae). Distr.-Brasil.

sp.1. Maia \& Couri, 1997: 229. Localidade: Brasil, RJ, Maricá. Hospedeiro: cecidomíideo (Diptera) galhador em Eugenia rotundifolia Casar (Myrtaceae); galha: enrolamento marginal da folha. Distr.-Brasil. Refs.: Maia (1995c); Maia \& Couri (1997).

sp.2. Maia \& Couri, 1997: 229. Localidade: Brasil, RJ, Maricá. Hospedeiro: cecidomíideo (Diptera) galhador em Myrciaria floribunda (West ex Willdenow) Berg. (Myrtaceae); galha: enrolamento marginal da folha. Refs.: Maia (1995c); Maia \& Couri (1997).

sp.3. Maia, 2001a. Localidade: Brasil, RJ, Maricá e Carapebus. Planta hospedeira: Stylosanthes guianensis Sw. (Leguminosae) - larvas livres na inflorescência. Distr.-Brasil.

sp.4. Maia, 2001a. Localidade: Brasil, RJ, Maricá. Planta hospedeira: Paullinia weinmanniaefolia Mart (Sapindaceae), galha: dobramento de folhas jovens. Distr.-Brasil.

\section{Tribo Lopesiini}

\section{Gênero Cordiamyia Maia}

Cordiamyia Maia, 1996a: 579, figs. 1, 5-10, 16-17. Espécie-tipo, globosa Maia (des. orig.).

globosa Maia, 1996a: 581, figs. 2-6, 8-18. Holótipo macho MNRJ, parátipos MNRJ. Localidade-tipo: Brasil, RJ, Maricá. Outras localidades: RJ, Arraial do Cabo e Carapebus. Planta hospedeira: Cordia verbenacea DC. (Boraginaceae).

\section{Gênero Lopesia Rübsaamen}

Lopesia Rübsaamen 1908: 29. Espécie-tipo, brasiliensis Rübsaamen (mon.).

brasiliensis Rübsaamen 1908: 30, figs. 11, 12. Síntipos, ZMHU. Localidade-tipo: Brasil, RJ, Rio de Janeiro; Santa Catarina, Tubarão. Planta hospedeira: Ossaea sp. (Melastomataceae). Distr-Brasil.

caulinaris Maia, 2002 (in Madeira et al., 2002): 32, figs 1-17b. Holótipo macho MNRJ, parátipos MNRJ. Localidade-tipo: Brasil, RJ, Carapebus. Planta hospedeira: Calophyllum brasiliense Camb. (Clusiaceae). Distr.-Brasil.

conspicua Maia, 2002 (in Madeira et al., 2002): 34, figs 18-34b. Holótipo macho MNRJ, parátipos MNRJ. Localidade-tipo: Brasil, RJ, Carapebus. Planta hospedeira: Calophyllum brasiliense Camb. (Clusiaceae). Distr.-Brasil. grandis Maia, 2001b: figs. 57-65. Holótipo macho, MNRJ, parátipos, MNRJ. Localidade-tipo: Brasil, RJ, Carapebus. Planta hospedeira: Dalbergia ecastophylla L. Taub (Leguminosae). Distr.-Brasil. 
elliptica Maia, 2002 (in Madeira et al., 2002): 37, figs 35-51b. Holótipo macho MNRJ, parátipos MNRJ. Localidade-tipo: Brasil, RJ, Carapebus. Planta hospedeira: Calophyllum brasiliense Camb. (Clusiaceae). Distr.-Brasil.

linearis Maia, 2002 (in Madeira et al., 2002): 41, figs 52-67b. Holótipo macho MNRJ, parátipos MNRJ. Localidade-tipo: Brasil, RJ, Carapebus. Planta hospedeira: Calophyllum brasiliense Camb. (Clusiaceae). Distr.-Brasil.

marginalis Maia, 2001b: figs. 66-74. Holótipo macho, MNRJ, parátipos, MNRJ. Localidade-tipo: Brasil, RJ, Maricá. Outras localidades: Rio de Janeiro, Arraial do Cabo. Planta hospedeira: Couepia ovalifolia (Schott) Benth (Chrysobalanaceae). Distr.-Brasil.

simplex Maia, 2002 (in Maia et al., 2002): figs. 14-26. Holótipo macho, MNRJ, parátipos, MNRJ. Localidade-tipo: Brasil, RJ, Carapebus. Planta hospedeira: Protium icicariba (DC.) March (Burseraceae). Distr.-Brasil.

singularis Maia, 2001b: figs. 75-81. Holótipo macho, MNRJ, parátipos, MNRJ. Localidade-tipo: Brasil, RJ, Maricá. Outras localidades: RJ, Arraial do Cabo. Planta hospedeira: Pouteria venosa (Mart.) Baehni (Sapotaceae). Distr.-Brasil.

\section{Gênero Myrciamyia Maia}

Myrciamyia Maia, 1995a: 567, figs. 1, 4-9. Espécietipo, maricaensis Maia (des. orig.).

maricaensis Maia, 1995a: 569, figs. 2-4, 6-14. Holótipo macho, MNRJ, parátipos MNRJ. Localidade-tipo: Brasil, RJ, Maricá. Outras localidades: RJ, Arraial do Cabo e Carapebus. Planta hospedeira: Myrcia ovata Camb. (Myrtaceae). Refs.: Maia (1995c); Maia \& Couri (1997).

\section{Gênero Rochadiplosis Tavares}

Rochadiplosis Tavares, 1917a: 33. Espécie-tipo, tibouchinae Tavares (des. orig.).

tibouchinae Tavares, 1917a: 34, figs. 5,6, pl. V, figs 1-10. Síntipos: parte presumivelmente perdida e parte na Coleção Felt. Localidade-tipo: Brasil, RJ, Nova Friburgo. Planta hospedeira: Tibouchina sp. (Melastomataceae). Distr.-Brasil.

Gêneros de Cecidomyiidi não posicionados em tribo

\section{Gênero Arrabidaeamyia Maia}

Arrabidaeamyia Maia, 2001b. Espécie-tipo, serrata Maia (des. orig.).

serrata Maia, 2001b: figs. 1-7. Holótipo macho MNRJ, parátipos MNRJ. Localidade-tipo: Brasil, RJ, Maricá. Outras localidades: RJ, Arraial do Cabo e Carapebus. Planta hospedeira: Arrabidaea conjugata (Vell.) Mart. (Bignoniaceae). Distr.-Brasil.

\section{Gênero Clusiamyia Maia}

Clusiamyia Maia, 1996b: 829, figs 1, 4-9. Espécie-tipo, nitida Maia (des. orig.).

nitida Maia, 1996b: 831, figs. 2-6, 8-10. Holótipo macho, MNRJ, parátipos MNRJ. Localidade-tipo: Brasil, RJ, Maricá. Outras localidades: RJ, Arraial do Cabo. Planta hospedeira: Clusia lanceolata Camb. (Clusiaceae). Distr.Brasil. Refs.: Maia (1999) e Maia (2001a).

granulosa Maia, 2001b: figs. 31-38. Holótipo macho, MNRJ, parátipos MNRJ. Localidade-tipo: Brasil, RJ, Carapebus. Planta hospedeira: Clusia hilariana Schltdl. (Clusiaceae). Distr.-Brasil.

\section{Gênero Contodiplosis Gagné}

Contodiplosis Gagné, 1994: 180. Espécie-tipo, Compsodiplosis friburgensis Tavares (des. orig.).

friburgensis Tavares, 1915: 150, figs. 3-5 (Compsodiplosis). Síntipos: presumivelmente perdidos; espécimens de ambos os sexos depositados na Coleção Felt, mas com etiqueta da Bahia. Localidade-tipo: Brasil, RJ, Nova Friburgo. Planta hospedeira: Styrax sp. (Styracaceae). Distr.-Brasil. Ref.: Gagné(1994).

humilis Tavares, 1915: 154, figs. 6-7 (Compsodiplosis). Síntipos: presumivelmente perdidos. Localidade-tipo: Brasil, RJ, Nova Friburgo. Planta hospedeira: Styrax sp. (Styracaceae). Distr.-Brasil. Ref.: Gagné (1994).

tristis Tavares, 1915: 156, fig. 8 (Compsodiplosis). Síntipos: presumivelmente perdidos. Localidade-tipo: Brasil, RJ, Nova Friburgo. Planta hospedeira: Styrax sp. (Styracaceae). Distr.-Brasil. Ref.: Gagné (1994).

\section{Gênero Dactylodiplosis Rübsaamen}

Dactylodiplosis Rübsaamen, 1916a: 452. Espécie-tipo, heisteriae Rübsaamen (mon.).

icicaribae Maia, 2002 (in Maia et al., 2002): figs. 113. Holótipo macho, MNRJ, parátipos MNRJ.

Localidade-tipo: Brasil, RJ, Carapebus. Planta hospedeira: Protium icicaribae(DC.) March (Burseraceae). Distr.-Brasil.

\section{Gênero Epihormomyia Felt}

Epihormomyia Felt, 1915a: 155. Espécie-tipo, auripes Felt (mon.).

miconiae Maia, 2001 b: figs. 47-56. Holótipo macho, MNRJ, parátipos MNRJ. Localidade-tipo: Brasil, RJ, Carapebus. Planta hospedeira: Miconia cinnamomifolia (DC.) Naudin (Melastomataceae). Distr.-Brasil.

\section{Gênero Frauenfeldiella Rübsaamen}

Frauenfeldiella Rübsaamen, 1905: 122. Espécie-tipo, coussapoae Rübsaamen (des. orig. como n. g. e n. sp.). 
coussapoae Rübsaamen, 1905: 122. Síntipos ZMHU. Localidade-tipo: Brasil, RJ, Rio de Janeiro (Gávea) e Amazonas (Juruá Miry). Planta hospedeira: Coussapoa sp. (Moraceae). Distr.-Brasil. Ref.: Gagné (1994).

\section{Gênero Karshomyia Felt}

Karshomyia Felt, 1908: 398. Espécie-tipo, Mycodiplosis viburni Felt (des. orig.).

Lobodiplosis Felt, 1908: 397. Espécie-tipo, Mycodiplosis acerina Felt (des. orig.).

sp. Localidade: Brasil, RJ, Niterói. Planta hospedeira: desconhecida. Distr.-Brasil.

\section{Gênero Manilkaramyia Maia}

Manilkaramyia Maia, 2001b. Espécie-tipo, notabilis Maia (des. orig.).

notabilis Maia, 2001b: figs. 82-88. Holótipo macho MNRJ, parátipos MNRJ. Localidade-tipo: Brasil, RJ, Carapebus. Planta hospedeira: Manilkara subsericea (Mart.) Dubard. (Sapotaceae). Distr.-Brasil.

\section{Gênero Mikaniadiplosis Gagné}

Mikaniadiplosis Gagné, 2001 (in Gagné et al., 2001). Espécie-tipo, annulipes Gagné (des. orig.).

annulipes Gagné, 2001 (in GAGNé, OdA \& MonteIro, 2001): figs.: 59-69. Holótipo macho MNRJ, parátipos MNRJ. Localidade-tipo: Brasil, RJ, Rio de Janeiro, Parati, Teresópolis e Silva Jardim (Reserva Biológica de Poço das Antas). Planta hospedeira: Mikania glomerata Sprengel (Asteraceae). Distr.-Brasil.

\section{Gênero Resseliella Seitner}

Resseliella Seitner, 1906: 174. Espécie-tipo, piceae Seitner (des. orig.).

sp.1. Maia, 1995a: 1003. Localidade: Brasil, RJ, Maricá. Planta-hospedeira: Eugenia rotundifolia Casar (Myrtaceae). Distr.-Brasil. Refs.: Maia (1995c: 1010); Maia \& Couri (1997).

sp.2. Maia, 2001a. Localidade: Brasil, RJ, Carapebus. Planta-hospedeira: Micania cinnamomifolia (DC) Naudin (Melastomataceae). Distr.-Brasil.

Novos registros de localidades apresentadas neste catálogo:

Arraial do Cabo: Alycaulus sp.; Arrabidaeamyia serrata; Asphondylia borreriae; Bruggmannia acaudata; Bruggmannia elongata; Bruggmannia robusta; Clinodiplosis profusa; Clusiamyia nitida; Cordiamyia globosa; Dasineura couepiae; Dasineura globosa; Lopesia marginalis; Lopesia singularis; Mayteniella distincta; Myrciamyia maricensis; Neolasioptera eugeniae; Neolasioptera sp.; Neomitranthella robusta; Parazalepidota clusiae; Paulliniamyia ampla; Proasphondylia guapirae; Stephomyia mina e Stephomyia rotundifoliorum.

Niterói: Dasineura globosa; Lopesia grandis; Karschomyia e Schismatodiplosis lantanae.

\section{AGRADECIMENTOS}

Agradeço a FAPERJ (Fundação de Amparo à Pesquisa do Estado do Rio de Janeiro, Proc. E-26/151.714/1999) pelo suporte financeiro: e a Luís Antônio Alves Costa (Museu Nacional/UFRJ) pelas ilustrações do adulto, larva e pupa.

\section{REFERÊNCIAS BIBLIOGRÁFICAS}

AGASSIZ, L. \& H. LOEW, 1846. Nomina systematica generum dipterorum. [Pt. 4] 42 pp. In Agassiz, L. Nomenclator Zoologicus. Fasc. 9/10: Titulum et praefationem operis, etc. Soloduri, Switzerland.

BARNES, H. F., 1946a. Gall Midges of Economic Importance. Vol. 1: Gall Midges of Root anf vegetable crops. Crosby Lockwood \& Son, London. 104 pp., 10 pls.

BARNES, H. F., 1946b. Gall Midges of Economic Importance. Vol. 2: Gall Midges of Fooder Crops. Crosby Lockwood \& Son, London. 160 pp., 3 pls.

BARNES, H. F., 1948a. Gall Midges of Economic Importance. Vol. 3: Gall Midges of Fruit. Crosby Lockwood \& Son, London. 184 pp., 9 pls.

BARNES, H. F., 1948b. Gall Midges of Economic Importance. Vol. 4: Gall Midges of Ornamental Plants and Shrubs. Crosby Lockwood \& Son, London. 165 pp., 10 pls.

BARNES, H. F., 1949. Gall Midges of Economic Importance. Vol. 6: Gall Midges of Miscellaneous Crops. Crosby Lockwood \& Son, London. 229 pp., 14 pls.

BARNES, H. F., 1951. Gall Midges of Economic Importance. Vol.5: Gall Midges of Trees. Crosby Lockwood \& Son, London. 270 pp., 8 pls.

BARNES, H. F., 1956. Gall Midges of Economic Importance. Vol. 7: Gall Midges of Cereal Crops. Crosby Lockwood \& Son, London. 261 pp., 16 pls.

COQUILLET, D. W., 1910. The Espécie-tipo of the North American genera of Diptera. Proceedings of United States Natural Musseum, Washington, D. C., 37: 499-647.

COURI M. S. \& V. C. MAIA, 1992. Considerações sobre Pisphondylia Möhn, 1960 (Diptera, Cecidomyiinae, Asphondyliidi), com descrição de uma espécie nova do Brasil. Revista brasileira de Entomologia , São Paulo, 36 (4): 729-730.

FELT, E. P., 1908. Appendix D. New York State Museum Bulletin, New York, 124: 286-422, pls. 33-44. 
FELT, E. P., 1915a. New South America gall midges. Psyche, Cambridge, 22: 152-157.

FELT, E. P., 1915b. New genera and species of gall midges. Proceedings of United States Museum, Washington, 48: 195-211.

FERNANDES, G. W. 1987. Gall Forming Insects: their economic importance and control. Revista Brasileira de Entomologia, São Paulo, 31 (3): 379-398.

GAGNÉ, R. J., 1968. [fascicle\} 23. Family Cecidomyiidae, pp. 1-62. In Papavero, ed. A Catalogue of the Diptera of the Americas South of the United States . Departamento de Zoologia, Secretaria da Agricultura, São Paulo, Brasil.

GAGNÉ, R. J., 1989. The Plant-Feeding Gall Midges of North America. Cornell University Press, Ithaca, N. Y. xi + 356 pp., 4 pls.

GAGNÉ, R. J., 1994. The Gall Midges of the Neotropical Region. Cornell University Press, Ithaca, N. Y. xiv + 352 pp.

GAGNÉ, R. J. 2004. A Catalog of the Cecidomyiidae (Diptera) of the World. Entomological Society of Washington, Washington, 408 pp.

GAGNÉ, R. J.; F. F. F. FERRAZ \& R. F. MONTEIRO, 1998. A new species of Neolasioptera Felt (Diptera: Cecidomyiidae) on Cuphea carthagenensis (Jacq.) Macbride (Lythraceae) in Brazil, with notes on its biology. Proceedings of Entomological Society of Washington, Washington, 100 (3): 521-525.

GAGNÉ, R. J.; R.A. M. ODA \& R. F. MONTEIRO, 2001. The gall midges (Diptera: Cecidomyiidae) of Mikania glomerata (Asteraceae) in southeastern Brazil. Proceedings of Entomological Society of Washington, Washington,

I. C. Z. N., 1958. International Trust for zoological Nomenclature, Londres, U.K..

KARSCH, F. A . F., 1877. Revision der Gallmücken. E. C. Brunn, Münster i. W. 58 pp., 1 pl.

KIEFFER, J. J.,1889. Neue Beiträge zur Kenntnis der Gallmücken. Entomologische Nachrichten, München, 15: 149-156, 171-176, 183-194, 202-212.

KIEFFER, J. J., 1894a. [untitled]. Bulletin Bimensuel de la Société Entomologique de France, Paris, 63: xxviii-xxix.

KIEFFER, J. J., 1894b. Description de quelques larves de Cécidomyes. Feuille des Jeunes Naturalistes, Paris, 24: 83-88, 119-121, 147-152, 185-189.

KIEFFER, J. J., 1895. [untitled]. Bulletin Bimensuel de la Société Entomologique de France, Paris, 63 (1894): cclxxx.

KIEFFER, J. J., 1901. Synopsis des zoocécidies d’ Europe. Annales de la Societé Entomologique de France, Paris, 70: 233-578.

KIEFFER, J. J., 1904. Nouvelles cécidomyies xylophiles. Annales de la Societé Scientifique de Bruxelles, Bruxelles, 28(1903): 367-410, pl.
KIEFFER, J. J., 1909. Contributions à la connaissance des insectes gallicoles. Bulletin de la Societé d'Histoire Naturelle de Metz, Metz, 28: 1-35.

KIEFFER, J. J., 1913. Diptera. Fam. Cecidomyiidae. Genera Insectorum, Bruxelles, 152: 1-346 pp., 15 pls.

KORYTKOWSKI, G. C. \& A . SARMIENTO, 1967. Hyperdiplosis sp. (Dipt.: Cecidomyiidae), un insecto formador de agallas en las hojas de la yuca. Revista Peruana de Entomologia, Lima, 10: 44-50.

LOEW, H., 1850. Dipterologische Beiträge. Vierter Teil. Oeffentl. Prüfung der Schüler des Königlichen FriedrichWilhelms-Gymnasiums zu Posen 1850, Berlin, 1-40.

MAIA, V. C., 1993a. Descrição de duas espécies novas de Cecidomyiidae (Diptera) associadas a Eugenia spp. (Myrtaceae). Revista Brasileira de Entomologia, São Paulo, 37 (4): 717-721.

MAIA, V. C., 1993b. Considerações sobre Proasphondylia Felt (Diptera, Cecidomyiidae) com descrições de duas espécies novas associadas com Guapira opposita (Vell.) Reitz. (Nyctaginaceae). Revista Brasileira de Zoologia, Curitiba, 10 (2): 215-218.

MAIA, V. C., 1993c. Considerações sobre Stephomyia Tavares (Diptera, Cecidomyiidae, Asphondyliidi), com descrição de quatro espécies novas associadas com Eugenia L. e Neomitranthes obscura (DC.) Legr. (Myrtaceae). Revista Brasileira de Zoologia, Curitiba, 10 (3): 521-530.

MAIA, V. C., 1993d. Uma nova espécie de Clinodiplosis Kieffer (Diptera, Cecidomyiidae) associada com Melissa officinalis Linnaeus (Labiatae) no Brasil. Revista Brasileira de Zoologia, Curitiba, 10 (4): 695-697.

MAIA, V. C., 1994. Myrciariamyia bivalva, gen. n. e sp. n. (Diptera, Cecidomyiidae) associado com Myrciaria floribunda (Camb.) Legr. (Myrtaceae) no Brasil. Revista Brasileira de Zoologia, Curitiba, 11 (4): 635-639.

MAIA, V. C., 1995a. Dois gêneros novos de Cecidomyiidae (Diptera) associados à Myrtaceae, na restinga da Barra de Maricá, Rio de Janeiro, Brasil. Revista Brasileira de Zoologia, Curitiba, 12 (3): 567-574.

MAIA, V. C., 1995b. Três espécies novas de Dasineura Rondani (Diptera, Cecidomyiidae) associadas a Myrtaceae na restinga da Barra de Maricá, Rio de Janeiro. Revista Brasileira de Zoologia, Curitiba, 12 (4): 1010-1008.

MAIA, V. C., 1995c. Chaves para a classificação de galhas de Cecidomyiidae (Diptera) em Myrtaceae na restinga da Barra de Maricá, Rio de Janeiro. Revista Brasileira de Zoologia, Curitiba, 12 (4): 1009-1013.

MAIA, V. C., 1996a. Cordiamyia globosa gen. n. e sp. n. (Diptera, Cecidomyiinae, Cecidomyiidi) associado com Cordia verbenacea DC. (Boraginaceae) no Brasil. Revista Brasileira de Zoologia, Curitiba, 13 (3): 579-583. 
MAIA, V. C., 1996b. Clusiamyia nitida gen. n. e sp. n. (Diptera, Cecidomyiinae, Cecidomyiidi) associado com Clusia lanceolata Camb. (Clusiaceae) no Brasil. Revista Brasileira de Zoologia, Curitiba, 13 (4): 829-832.

MAIA, V. C., 1999. Artrópodes associados às galhas de Cecidomyiidae (Diptera) em Eugenia, rotundifolia (Myrtaceae) e Clusia lanceolata (Clusiaceae) em uma restinga do Rio de Janeiro, Brasil. Iheringia, Série Zoológica, Porto Alegre, 87: 75-79.

MAIA, V. C., 2001 a. The gall midges (Diptera, Cecidomyiidae) from three restingas of Rio de Janeiro State, Brazil. Revista Brasileira de Zoologia, Curitiba, 8 (2): 583-630.

MAIA, V. C., 2001 b. New genera and species of gall midges (Diptera, Cecidomyiidae) from three restingas of Rio de Janeiro State, Brazil. Revista Brasileira de Zoologia, Curitiba, 18(Supl. 1): 1-32.

MAIA, V. C., 2001 c. Two new species of gall midges (Diptera, Cecidomyiidae) assocciated with Pouteria caimito var. laurifolia (Sapotaceae) in Brazil. Studia Dipterologica, Saale, 8 (1): 103-110.

MAIA, V. C. 2004. Description of a new species of Bruggmannia Tavares (Diptera, Cecidomyiidae) associated with Guapira opposita (Vell.) Reitz. (Nyctaginaceae). Revista Brasileira de Zoologia, Curitiba, 21(4): 761-764.

MAIA, V. C. \& M. S. COURI, 1992. Descrição de três espécies novas de Bruggmannia Tavares, 1906 (Diptera, Cecidomyiidae, Asphondyliidi) do Brasil associadas com Guapira opposita (Nyctaginaceae). Revista Brasileira de Biologia, Rio de Janeiro, 53 (2): 209-215.

MAIA, V. C. \& M. S. COURI, 1997. Cecidomyiidae (Diptera) associated with Myrtaceae at restinga of Barra de Maricá (RJ). Revista Brasileira de Biologia, Curitiba, 57 (2): 227-230.

MAIA, V. C.; M. S. COURI \& R. F. MONTEIRO, 1992. Sobre seis espécies de Asphondylia Loew, 1850 do Brasil (Diptera, Cecidomyiidae). Revista Brasileira de Entomologia, São Paulo, 36 (3): 653-661.

MAIA,V.C. \& R. F. MONTEIRO, 1999. Espécies cecidógenas (Diptera, Cecidomyiidae) e parasitóides (Hymenoptera) associadas a Guapira opposita (Vell.) Reitz. (Nyctaginaceae) na restinga da Barra de Maricá. Revista Brasileira de Zoologia, Curitiba, 16 (2): 483-487.

MAIA, V. C.; R. F. MONTEIRO \& K. L. NARAHARA, 2002. Two new species of gall midges (Diptera, Cecidomyiidae) associated with Protium icicariba (Burseraceae) in Brazil. Studia Dipterologica, Saale, 9 (1): 171-178.

MADEIRA, J. A.; V. C. MAIA \& R. F. MONTEIRO, 2002. Gall makers (Diptera, Cecidomyiidae) on Calophyllum brasiliense Camb. (Clusiaceae): descriptions and biology. Arquivos do Museu Nacional, Rio de Janeiro, 61(1):31-48.
MÖHN, E., 1959. Gallmücken (Diptera, Itonididae) aus El Salvador. 1. Teil. Senckenbergiana Biologica, Frankfurt, 40: 297-368.

MÖHN, E., 1960. Gallmücken (Diptera, Itonididae) aus El Salvador. 2. Teil. Senckenbergiana Biologica, Frankfurt, 41: 197-240.

MÖHN, E., 1961. Neue Asphondyliidi-Gattungen (Diptera, Itonididae). Stuttgarter Beiträge zur Naturkunde, Stuttgart, 49: 1-14.

MÖHN, E., 1962. Studien über neotropische Gallmücken (Diptera, Itonididae).1.Teil. Broteria, Série de Ciências Naturais, Braga, 31: 211-239.

MÖHN, E., 1963. Studien über neotropische Gallmücken (Diptera, Itonididae).1.Teil. (Fortsetzung). Broteria, Série de Ciências Naturais, Braga, 32: 3-23.

MÖHN, E., 1964. Gallmücken (Diptera, Itonididae) aus El Salvador. 7. Teil, Lasiopteridi. Beiträge zur Entomologie, Berlin, 14: 553-600.

MÖHN, E., 1973. Studien über neotropische Gallmücken (Diptera, Itonididae). 2. Teil. Stuttgarter Beiträge zur Naturkunde (A), Stuttgart, 257: 1-9.

MÖHN, E., 1975. Gallmücken (Diptera, Itonididae) aus El Salvador. 8. Teil, Lasiopteridi. Stuttgarter Beiträge zur Naturkunde(A), Stuttgart, 276: 1-101.

NIJVELDT, W. C. 1969. Gall Midges of Economic Importance. Vol. VIII: Gall Miscellaneous. Crosby Lockwood \& Son, London. 221 pp., 12 pls.

PAMPLONA, D. M.; V. C. MAIA; M. S. COURI; C. J. E. LAMAS \& C. C. DE C. AIRES, 2000. A survey on Diptera of Paquetá Island, Rio de Janeiro, RJ (Insecta, Diptera). Entomologist’s Monthly Magazine, London, 136: 169-175.

RONDANI, C. , 1840. Memoria per servir alla ditterologia italiana. No. 2: sopra alcuni nuovi generi di insetti ditteri. Parma, Parma, 28 pp., 1pl.

RONDANI, C., 1846. Compendio della seconda memoria ditterologica (publicata 1840) com algune aggiunte et correzioni. Nuovi Annali delle Scienze Naturali di Bologna, Bologna, 6: 363-376, 1 pl.

RONDANI, C., 1956. Dipterologiae Italicae prodomus. Vol. 1: Genera Italica ordinis dipterorum ordinatum disposita et distincta et in familias et stires agregata. Parma, Parma, 228 pp.

RONDANI, C., 1860. Stirpis cecidomyarum. Genera revisa. Noota undecima, pro dipterologia italica. Atti della Societá Italiana di Scienze Naturali (1859-1860), Milano, 2: 286-294, 1 pl.

RÜBSAAMEN, E. H., 1892: Die Gallmücken des Königl. Museums für Naturkunde zu Berlin. Berliner Entomologische Zeitschrift, Berlin, 37: 319-411, pls. VII-XVIII. 
RÜBSAAMEN, E. H., 1905. Beiträge zur Kenntnis aussereuropäischer Zoocecidien. II. Beitrag: Gallen aus Brasilien und Peru. Marcellia, Avellino, 4: 65-85, 115-138.

RÜBSAAMEN, E. H., 1907. Beiträge zur Kenntnis aussereuropäischer Zoocecidien. III. Beitrag [cont.]: Gallen aus Brasilien und Peru. Marcellia, Avellino, 6: 110-173.

RÜBSAAMEN, E. H., 1908. Beiträge zur Kenntnis aussereuropäischer Zoocecidien. III. Beitrag: Gallen aus Brasilien und Peru. Marcellia , Avellino, 7: 15-79.

RÜBSAAMEN, E. H., 1916a. Beitrag zur Kenntnis aussereuropäischer Gallmücken. Sitzungsberichte der Gesellshaft Naturforschender Freunde zu Berlin 1915, Berlin, 431-485.

RÜBSAAMEN, E. H., 1916b. Cecidomyidenstudien V. Revision der deutschen Asphondylarien. Sitzungsberichte der Gesellschaft Naturforschender Freunde zu Berlin, Berlin, 1916: 1-12.

SEITNER, M., 1906. Resseliella piceae, die Tannensamengallmücke. Verhandlungen der zoologisch-botanischen Gesellschaft in Wien 56, Wien: 174-186.

SKUHRAVÁ, M., 1989. Taxonomic changes and records in palaeartic Cecidomyiidae (Diptera). Acta Entomologica Bohemoslovaca, Praha, 86: 202-233.

TAVARES, J. S., 1906. Descripção de uma Cecidomyia nova do Brazil, pertencente a um genero novo. Broteria, Braga, 5: 81-84.

TAVARES, J. S., 1909. Contributio prima ad cognitionem cecidologiae Braziliae. Broteria, Série Zoológica, Braga, 8: 5-28, pls. I-VIII.

TAVARES, J. S., 1915. As cecidias das plantas do genero Styrax no Brazil. Broteria, Série Zoológica, Braga, 13: 145-160, pl. VI.

TAVARES, J. S., 1916. Cecidomyias novas do Brazil. Broteria, Série Zoológica, Braga, 14: 36-57.

TAVARES, J. S., 1917a. As cecidias do Brazil que se criam nas plantas da familia das Melastomataceae. Broteria, Série Zoológica, Braga, 15: 18-49, pls. I-V.

TAVARES, J. S., 1917b. Cecidias brazileiras que se criam em plantas das Compositae, Rubiaceae, Tiliaceae, Lythraceae e Artocarpaceae. Broteria, Série Zoológica, Braga, 15: 113-181, pls. VI-XI.

TAVARES, J. S., 1918a. Cecidologia brazileira. Cecidias que se criam em plantas das familias das verbenaceae, Euphorbiaceae, Malvaceae, Anacardiaceae, Labiatae, Rosaceae, Anonaceae, Ampelidaceae, Bignoniaceae, Aristolochiaceae e Solanaceae. Broteria, Série Zoológica, Braga, 16: 21-68, pls. I-II.

TAVARES, J. S., 1918b. Cecidomyias novas do Brazil. Segunda série. Broteria, Série Zoológica , Braga, 16: 6984, pls. III-IV.
TAVARES, J. S., 1920a. O gênero Bruggmannniella Tav. Com descripção de uma espécie nova e a clave dichotômica dos gêneros das Asphondyliariae. Broteria, Série Zoológica, Braga, 18: 33-42.

TAVARES, J. S., 1920b. Cecidologia brazileira. Cecidias que se criam em plantas das familias das Leguminosae, Sapotaceae, Lauraceae, Myrtaceae, Punicaceae, Aurantiaceae, Malpighiaceae, Sapindaceae, Umbelliferae, Loranthaceae, Apocynaceae, Urticaceae, Salicaceae e Graminae. Broteria, Série Zoológica, Braga, 18: 82-125, pl. III.

TAVARES, J. S., 1920c. Anadiplosariae, nouvelle tribu de Cecidomyiidae (Dipt). Marcellia, Avellino, 17: 57-72, pl. I.

TAVARES, J. S., 1921. Cecidologia brazileira. Cecidias que se criam em plantas das familias das Leguminosae, Sapotaceae, Lauraceae, Myrtaceae, Punicaceae, Aurantiaceae, Malpighiaceae, Sapindaceae, Umbelliferae, Loranthaceae, Apocynaceae, Urticaceae, Salicaceae e Graminae. Broteria, Série Zoológica, Braga, 19: 76-112, pl. 1.

TAVARES, J. S., 1922. Cecidologia brazileira. As restantes famílias. 26:385 394, 25 figs. Broteria, Série Zoológica,Braga, 20: 5-48c, pls. XI-XIX.

Título: Catálogo dos Cecidomyiidae (Díptera) do Estado do Rio de Janeiro.

Autor: Valéria Cid Maia

Biota Neotropica, Vol. 5 ( número 2): 2005

http://www.biotaneotropica.org.br/v5n2/pt/ abstract?inventory+BN00805022005

Recebido em 10/12/04.

Revisado em 16/04/05.

Publicado em 01/07/2005.

ISSN 1676-0603 